\title{
Address2vec: Generating Vector Embeddings for Blockchain Analytics
}

Ali Hussein, Samiiha Nalwooga

MAKERERE UNIVERSITY

Ronin Institute - Artificial Intelligence and Data Science Research Lab, Makerere University

\section{Objectives}

1. Generating dense vector embeddings that encode the semantic relationships in the bitcoin blockchain.

2. Testing generated embeddings against a baseline approach

\section{Introduction}

- Bitcoin is a virtual currency that has a completely open transaction ledger

- Bitcoin blockchain is designed for security not analysis

- Addresses are unique on the Bitcoin Blockchain

- Bitcoin addresses are used to "hoard" or "spend" bitcoins

\section{Methods}

\section{- Bitcoin Transaction Graph}

$\triangleright$ I query the blockchain.org API for block data (blocks 200,000-200,100) and all transaction histories for all output addresses in the blocks.

$\triangleright$ I use networkX to transform each address into a graph node and each transaction into a weighted directed edge.

$\triangleright \mathrm{I}$ use an autoencoder to compress the features in the feature space into a single value which is put as a weight in the directed transaction edges

- Word2vec

$\triangleright$ An efficient method to generate dense vectors that encode the semantic and syntactic relationships between words in a corpus.

$\triangleright$ Each word has a generated embedding and relationships

(synonimity/antonimity) between words can be deduced by measuring their vectors cosine distance.

$\checkmark$ Node2vec

$\triangleright$ Extends the word2vec model to generate embeddings for nodes in a graph

$\triangleright$ I use node2vec to generate embeddings for each node/address in the Bitcoin Transaction Graph.

\section{Visualization of Bitcoin Transaction Graph}

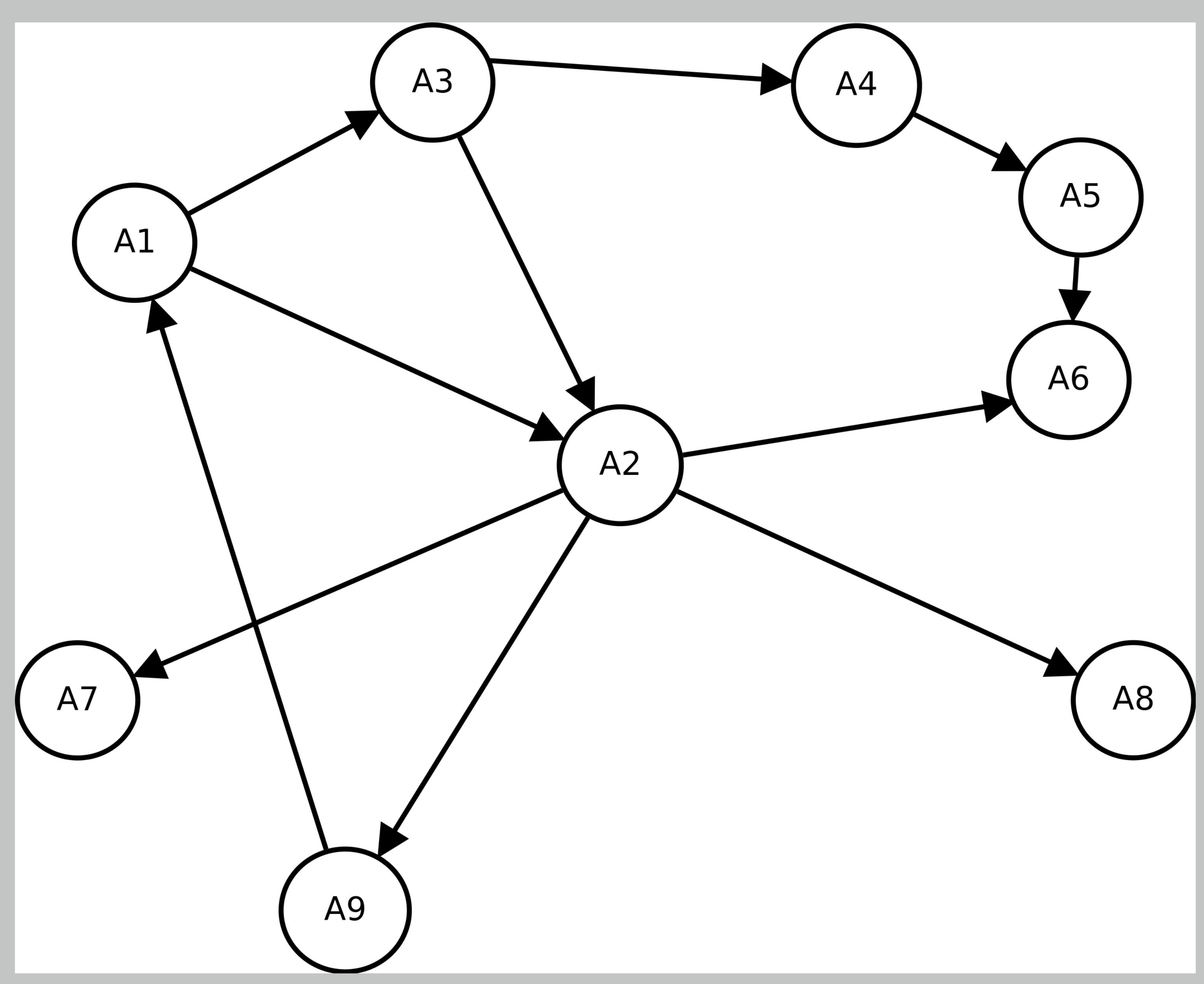

Figure 1: This is a an example of a transaction graph where each node $\mathrm{A}$ is a bitcoin address and each weighted edge is a transaction between 2 addresses where one is sending and the other receiving and the weight is the amount transacted in bitcoin
Visualization of Bitcoin Address Embeddings

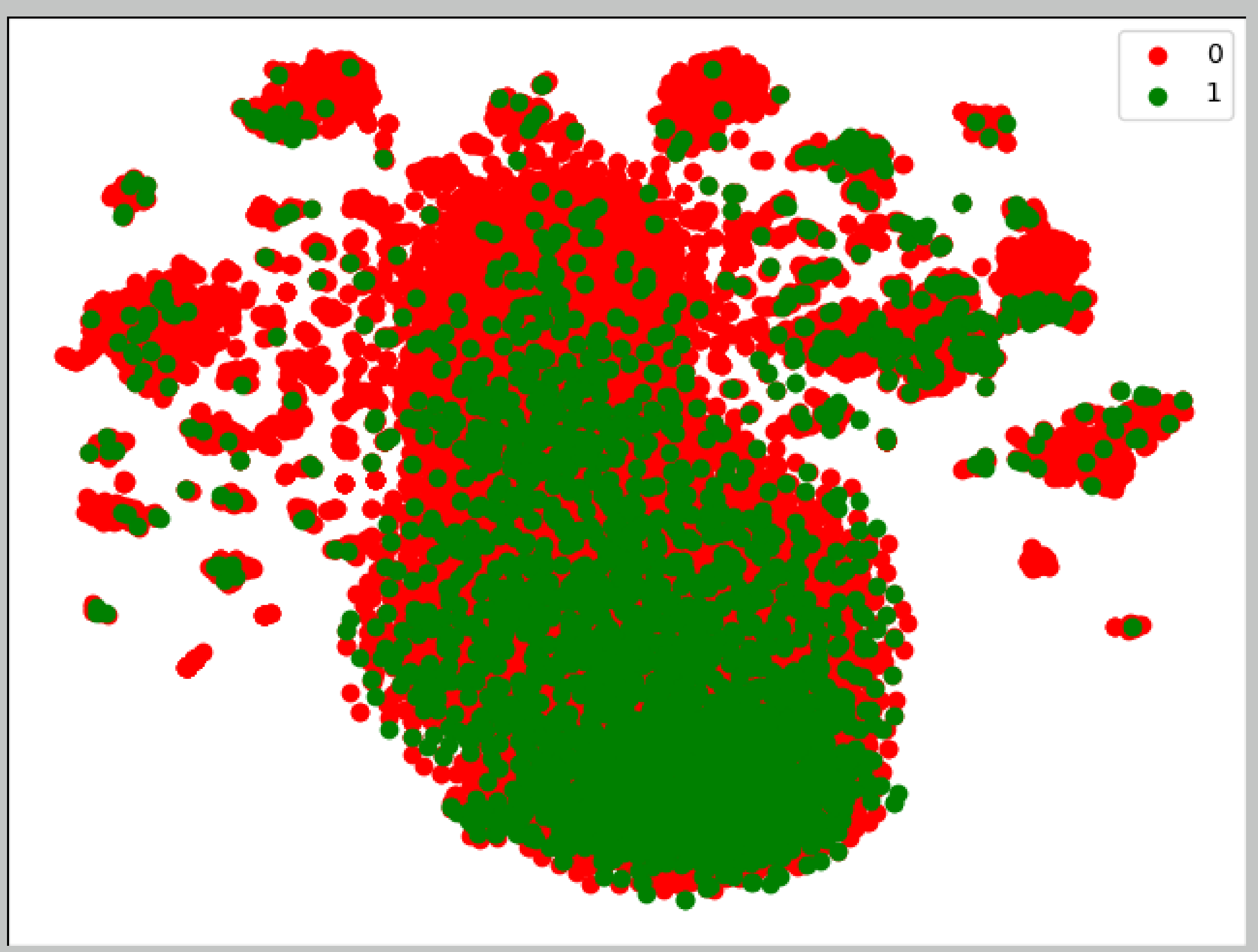

Figure 2: This is a t-SNE reduction of the blockchain embeddings, we see distinct groups of spending on the edges but overall spending and hoarding behaviours seem to occur in the same clusters with hoarding more common

\section{Experiment Design}

- A baseline model that predicts the liklehood of a class based on its incidence (a biased coinflip).

- Embeddings Generated at various hyperparameter values are stored and seed a single hidden layer feed forward Neural Network with a sigmoid output layer.

\section{Results: Table}

- Results of predicting hoarding vs spending status for bitcoin addresses

\begin{tabular}{lll}
\hline \multicolumn{4}{c}{ Avg Recall F1 (Spent) } & F-1 (Hoard) \\
\hline Baseline 0.50 & 0.02 & 0.66 \\
1-MLSL 0.79 & 0.81 & 0.62 \\
2-MLSL 0.79 & 0.85 & 0.63 \\
3-MLSL 0.78 & 0.84 & 0.63 \\
A2V $\quad 0.68$ & 0.79 & 0.43
\end{tabular}

Table 1: Bitcoin hoarding prediction results using address2vec embeddings in a Densely connected NN vs a baseline approach

- Our embedding approach is slightly better than the baseline model.

- A2V performs consistently worse than Multiple Level Sequepnce Learners

\section{Conclusion}

- The results reached show promise in adapting blockchain embeddings to many domains.

- Further experiments and tuning are needed

\section{Future Work}

- Testing prediction of bitcoin price instability

- Classfying bitcoin addresses related to certain behaviours ex. blackmarket activities.

\section{Acknowledgments}

- Thanks to Benjamin Akera, Mohamed Emad, Shahinaz Gamal, Jens Mucke, Randa Fahmy, Jon F Wilkins and Octavio Paez Osuna 